\begin{tabular}{|c|l|}
\hline Title & Spectrum of laser light scattered by nanoparticles in an abl ation-induced cavitation bubble \\
\hline Author(s) & Takeuchi, Masato; Sasaki, Koichi \\
\hline Citation & $\begin{array}{l}\text { A pplied physics A : Materials science \& processing, 122(4), 312 } \\
\text { https://doi.org/L0.1007/300339-016-9927-8 }\end{array}$ \\
\hline Issue Date & 2016-04 \\
\hline Doc URL & http://hdl.handle.net/2115/64912 \\
\hline Rights & The original publication is available at www.springerlink.com \\
\hline Type & article (author version) \\
\hline File Information & CoLA_2015_APA_HUSCAP.pdf \\
\hline
\end{tabular}

Instructions for use 


\title{
Spectrum of laser light scattered by nanoparticles in an ablation-induced cavitation bubble
}

\author{
Masato Takeuchi · Koichi Sasaki
}

Received: date / Accepted: date

\begin{abstract}
The spectrum of the laser light scattered by nanoparticles in a cavitation bubble, which was induced by laser ablation of a titanium target in water, was measured using a triple-grating spectrograph. The scattered laser light observed at $100 \mu$ s after laser ablation had no wavelength-shifted component, suggesting that nanoparticles at this delay time were metallic. The wavelength-shifted component was observed in the spectrum at a delay time of $200 \mu \mathrm{s}$, suggesting the formation of oxidized nanoparticles. However, we observed no peaks in the spectrum of the scattered laser light in the present in-situ laser-light scattering experiment. On the other hand, we observed clear peaks in the Raman spectrum of synthesized nanoparticles. The experimental results suggest slow crystallization of nanoparticles in liquid in liquid-phase laser ablation.
\end{abstract}

Keywords in-situ Raman scattering · liquid-phase laser ablation · synthesis of nanoparticles $\cdot$ titanium dioxide

\section{Introduction}

Liquid-phase laser ablation attracts much attention as a new method for synthesizing functional nanoparticles [1-4]. This method has a technical advantage that the change of the target and the collection of nanoparticles are easier in comparison with other methods using vacuum equipment. In addition, liquidphase laser ablation has particular feature from the physical point of view. The plasma produced by irradiating an intense laser pulse onto a solid target in liquid has a high pressure and a high temperature [5,6]. The high-pressure,

Division of Quantum Science and Engineering, Hokkaido University, Kita 13, Nishi 8, Kita$\mathrm{ku}$, Sapporo 060-8628, Japan

Tel.: +81-11-706-6654

Fax: +81-11-706-6655

Email: sasaki@qe.eng.hokudai.ac.jp 
high-temperature plasma induces the formation of a cavitation bubble. The cavitation bubble has the dynamics of the expansion and the shrinkage, and the shrinkage of the cavitation bubble is followed by the collapse [7-9]. It is known that another high-pressure, high-temperature state is realized by the collapse of the cavitation bubble [10]. The high-pressure, high-temperature reaction fields work helpfully in the synthesis of nanoparticles at high-pressure and metastable phases [11].

A problem of liquid-phase laser ablation is the insufficient understanding on fundamental processes of the nanoparticle formation. The first event in liquidphase laser ablation is the ejection of atoms and molecules from the target into the liquid. The ejection of atoms and molecules is followed by the formation of a cavitation bubble. Hence it is sure that the particles ejected from the target are located outside of the cavitation bubble in the very initial phase. Since the cavitation bubble expands with time, the boundary of the cavitation bubble reaches the region with ejected particles at a later delay time. Here a fundamental question arise about the growth place of nanoparticles: in liquid or in the cavitation bubble.

We have given an answer to this question by carrying out simultaneous imaging of shadowgraph and laser-light scattering [12]. The experimental result clearly shows the growth of nanoparticles inside the cavitation bubble. The growth of nanoparticles inside the cavitation bubble has been confirmed by Ibrahimkutty and coworkers by small-angle x-ray scattering $[13,14]$. Another important knowledge obtained by our previous experiment is that nanoparticles are stored inside the cavitation bubble until its collapse. Nanoparticles are located in the high-pressure, high-temperature reaction field at the collapse of the cavitation bubble. We have demonstrated the control of the size and the structure of nanoparticles by controlling the dynamics of the cavitation bubble [15-17]. It is widely understood now that the cavitation bubble plays an important role in the synthesis of nanoparticles by liquid-phase laser ablation [18-20].

In this work, we measured the spectrum of the laser light scattered by nanoparticles inside the cavitation bubble. The scattered laser light has no wavelength-shifted component if nanoparticles are metallic, while we may observe the scattered laser light with the wavelength shift if nanoparticles are Raman active. In addition, the spectrum of the scattered laser light has peaks if nanoparticles have crystalline structures. The laser-light scattering experiment reported in the previous work gave us the information on the temporal evolution of the number of nanoparticles, while the present work is an attempt to understand the qualitative change of nanoparticles inside the cavitation bubble.

\section{Experiment}

The experimental apparatus is schematically shown in Fig. 1. We prepared a rectangular vessel with a volume of $360 \mathrm{~cm}^{3}$ for this experiment. The vessel 
had five optical windows with a thickness of $1 \mathrm{~mm}$ on its four sides and the top. A titanium target was inserted into the vessel from the bottom. The target was rotated in the vessel using a motor to disperse the ablation point. The vessel was filled with distilled water, and the water surface attached to the top window. We used two Nd:YAG lasers at the second harmonics (532 $\mathrm{nm}$ ) in this experiment. One was used for ablating the Ti target. The laser beam for ablation was introduced into the vessel via the top window. A lens was used for focusing the laser beam onto the target surface. The energy of the laser beam for ablation was $100 \mathrm{~mJ} /$ pulse, corresponding to the fluence of approximately $2.5 \mathrm{~J} / \mathrm{cm}^{2}$ on the target surface. The other YAG laser was used for the scattering experiment. The laser beam for scattering was injected into the vessel via the side window. It was focused using a lens with a focal length of $250 \mathrm{~mm}$ onto the position above the ablation point. The energy of the laser beam for scattering was $10 \mathrm{~mJ} /$ pulse. The distance between the laser beam and the target surface was roughly $3 \mathrm{~mm}$. The laser beam transmitted through the vessel was blocked using a beam dump. Both the two YAG lasers were operated at repetition frequencies of $10 \mathrm{~Hz}$, and the delay time between the oscillations was controlled using a pulse generator.

The scattered laser light was collimated using a lens with a focal length of $250 \mathrm{~mm}$ and a diameter of $100 \mathrm{~mm}$. The collimated light was focused onto the entrance slit of a triple grating spectrograph using a lens with a focal length of $200 \mathrm{~mm}$. The image beam of the scattered laser light was tilted using two aluminum mirrors, and the path of the laser beam for scattering was projected onto the entrance slit of the triple grating spectrograph. The triple grating spectrograph, which was developed for a laser Thomson scattering experiment [21], was equipped with three diffractive gratings (1800 grooves/mm), six lenses, a spatial filter (called "Rayleigh block"), an intermediate slit, and a charge coupled device camera with a gated image intensifier (ICCD camera). The focal lengths of the lenses in the spectrograph were $200 \mathrm{~mm}$. The Rayleigh block was an important component which worked for eliminating the scattered laser light at the laser wavelength very efficiently [22,23]. In this optical configuration, the $x$-axis in the image recorded using the ICCD camera corresponded to the wavelength, while the $y$-axis corresponded to the position along the path of the laser beam for scattering. The gate opening of the ICCD camera was synchronized with the laser pulse for scattering. We accumulated the signals for 200 laser shots to compensate the poor shot-to-shot reproducibility.

We used a cw laser at $532 \mathrm{~nm}$, which is not illustrated in Fig. 1, as the back light of shadowgraph imaging. The third laser was placed on the opposite side of the triple grating spectrograph (the backside of Fig. 1). The laser beam irradiated a scatterer sheet, and the ablation space was illuminated by the dispersive laser light from the beam scatterer. When the entrance and intermediate slits in the triple grating spectrograph were wide enough (typically 10 $\mathrm{mm}$ ), the triple grating spectrograph did not work as a spectrometer and it transmitted the shadowgraph image of the ablation space to the ICCD camera. In this way, we recorded the shadowgraph image of the cavitation bubble 


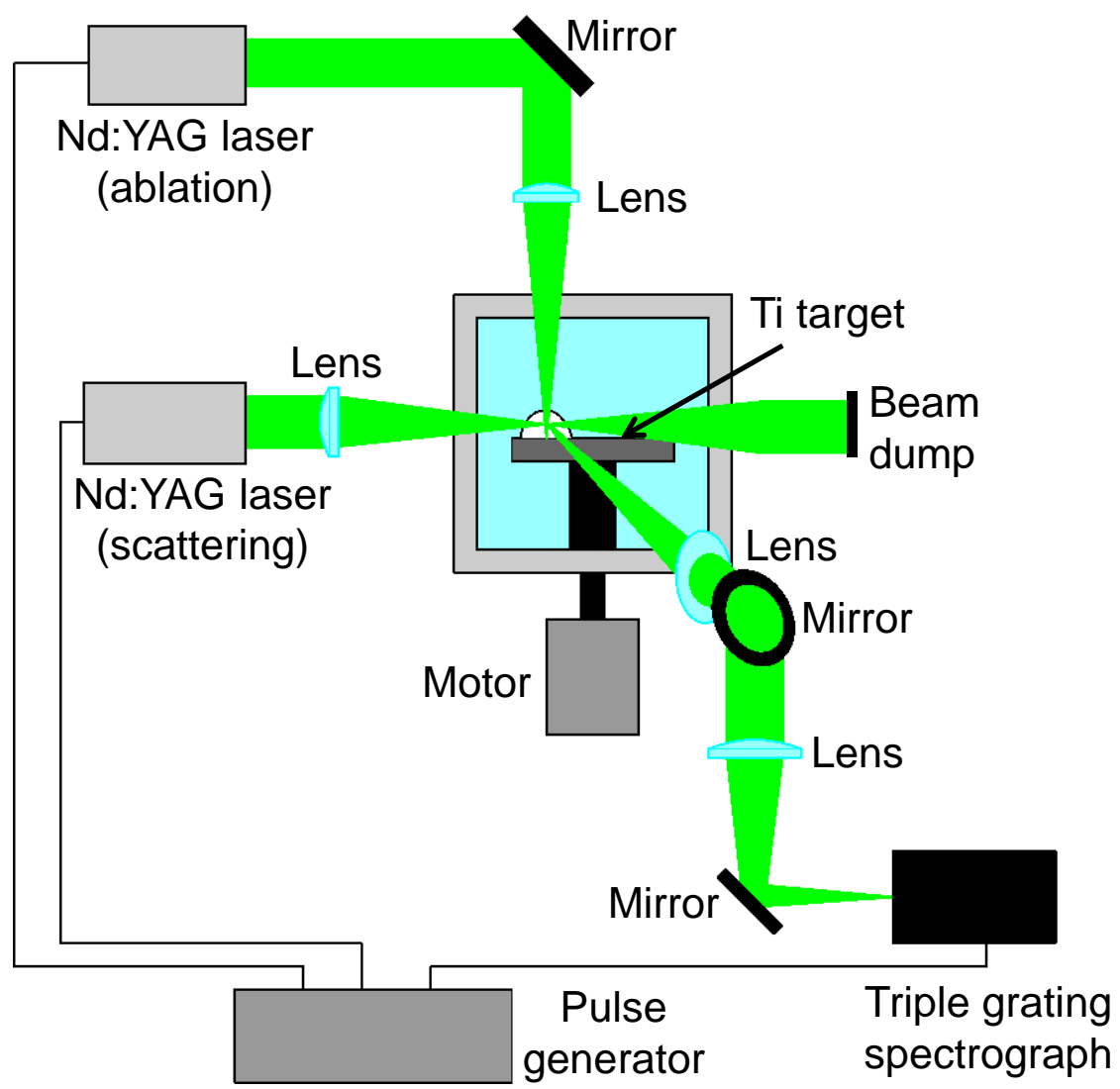

Fig. 1 Experimental apparatus.

with keeping the optical alignment for the laser light scattering experiment. The YAG laser for scattering was switched off in the shadowgraph imaging, while the cw laser was switched off in the scattering experiment.

The experimental apparatus shown in Fig. 1 had difficulty in the fine focusing of the incident laser beam for scattering and the collimation of the scattered laser light. This is because the incident laser beam and the image beam of the scattered laser light passed through two gas/liquid boundaries. One boundary was the interface between air (the outside of the vessel) and water (the inside of the vessel). Here we ignored the influence of the optical window by considering its thickness $(1 \mathrm{~mm})$. The other boundary was the interface between water and the cavitation bubble. According to optical ray tracing, the focal points of the laser and image beams are located above the ablation point when the distance between the focusing (or collimating) lens 
and the edge of the vessel is adjusted such that

$$
L=f-\frac{d-2 R}{n},
$$

where $n$ is the refractive index of water, $f$ is the focal length of the focusing (or collimating) lens, and $d$ is the distance between the edge of the vessel and the ablation point. The bubble was assumed to be hemispherical one with radius $R$, and the refractive index inside the cavitation bubble is assumed to be unity. Since the bubble size changed temporally, the positions of the lenses were adjusted according to the delay time between the oscillations of the two YAG lasers.

\section{Results}

First of all, we measured the spectrum of the scattered laser light when the YAG laser for ablation and the backlight laser were switched off. No cavitation bubbles were formed in this case. When the Rayleigh block was removed and the widths of the entrance and intermediate slits were sufficiently wide, we observed the fine image of the scattered laser light by adjusting the position of the collimating lens. When the Rayleigh block was inserted and the slit widths were adjusted at $50 \mu \mathrm{m}$, the picture shown in Fig. 2(a) was recorded on the ICCD camera. The region corresponding to the Rayleigh block was masked in Fig. 2(a). The horizontal axis of Fig. 2(a) shows the shift from the laser wavelength, while the vertical axis shows the position along the path of the laser beam. The strong scattered laser light, which was caused by Rayleigh scattering, was observed at the laser wavelength, but it was blocked efficiently with the help of the Rayleigh block. The weak scattered laser light was observed around the laser wavelength as shown in Fig. 2(a). Figure 2(b) shows the spectrum of the scattered laser light, which was obtained by integrating the picture shown in Fig. 2(a) along the vertical direction, together with the Raman spectrum of liquid water [24,25]. The close agreement shown in Fig. 2(b) indicates that the apparatus shown in Fig. 1 works for the in-situ measurement of the Raman spectrum in the ablation space.

Figure 3 shows the shadowgraph images (left) and the spectra of the scattered laser light (right), which were observed at three delay times after laser ablation. The vertical shadows in the shadowgraph images are caused by the Rayleigh block. A relatively large cavitation bubble was induced in this experiment to inject the laser beam for scattering into the cavitation bubble easily. The images shown in Fig. 3(a) were observed at a delay time of $t=100 \mu \mathrm{s}$, which corresponded to the expansion phase of the cavitation bubble. As shown in Fig. 3(a), we observed the Raman scattering of water from the outside region of the cavitation bubble. The observation of the Raman scattering of water in the both sides of the cavitation bubble indicates that the laser beam for scattering passed through the cavitation bubble. It is noted that the scattered laser light was also observed from the region corresponding to the inside of 


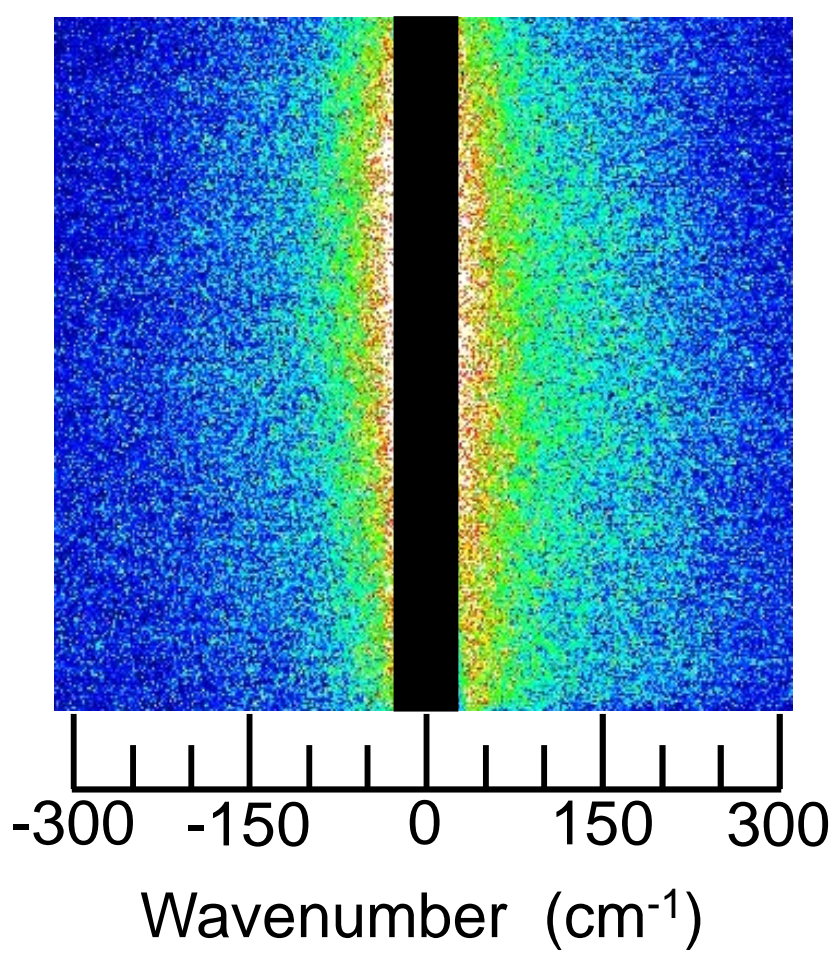

(a)

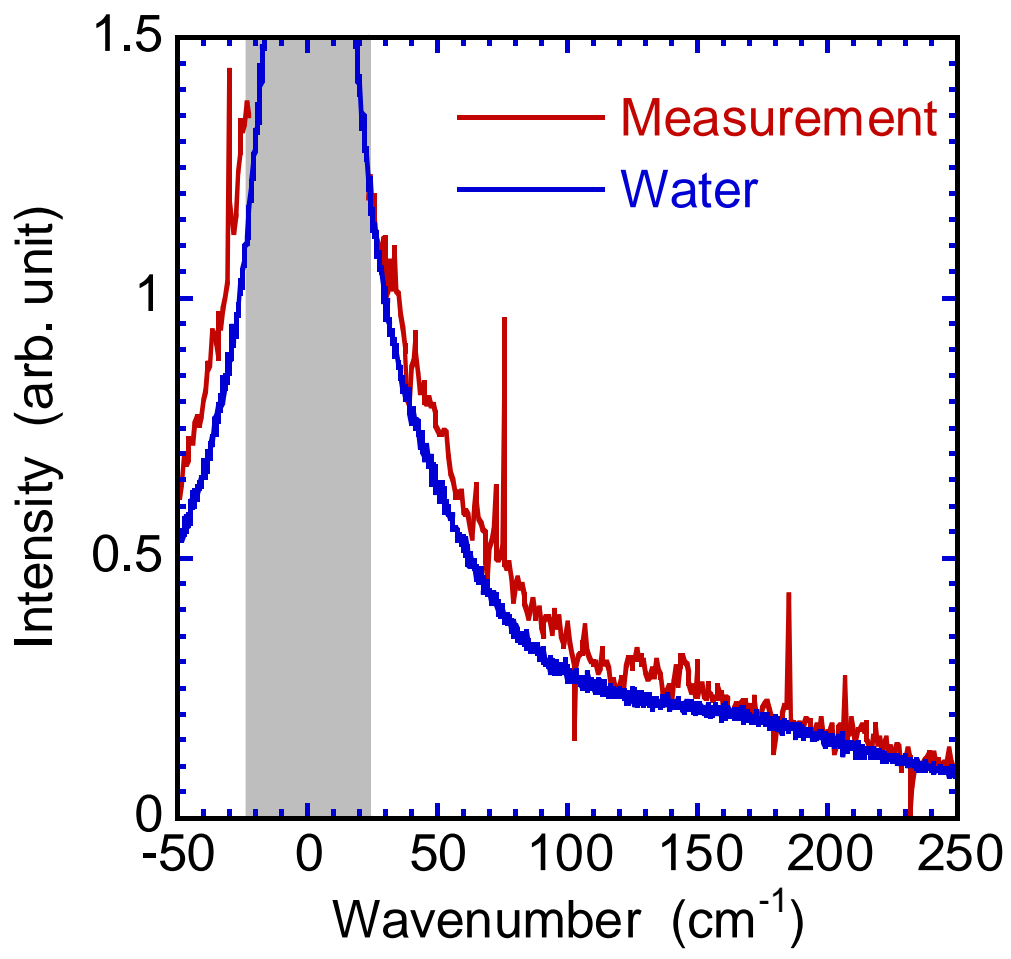

(b)

Fig. 2 (a) Image captured using the ICCD camera in the absence of the YAG laser for ablation. The horizontal axis shows the shift from the laser wavelength, and the vertical axis corresponds to the position along the path of the laser beam. (b) Spectrum of the scattered laser light, which was obtained by integrating the image shown in (a) along the vertical direction. 

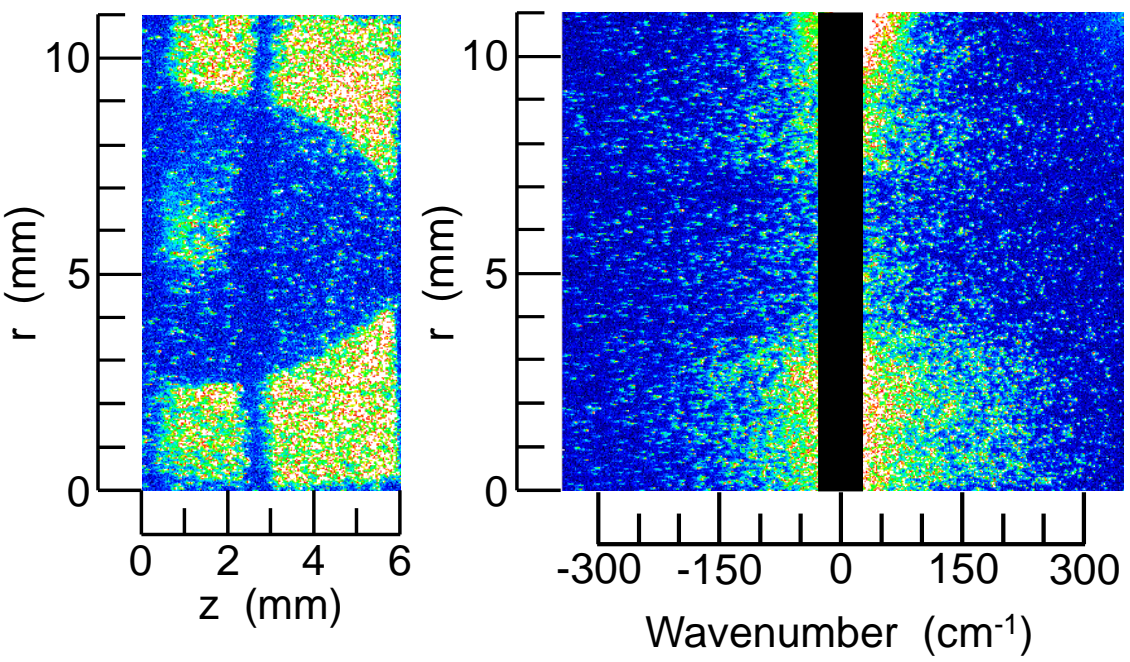

(a) $t=100 \mu \mathrm{s}$
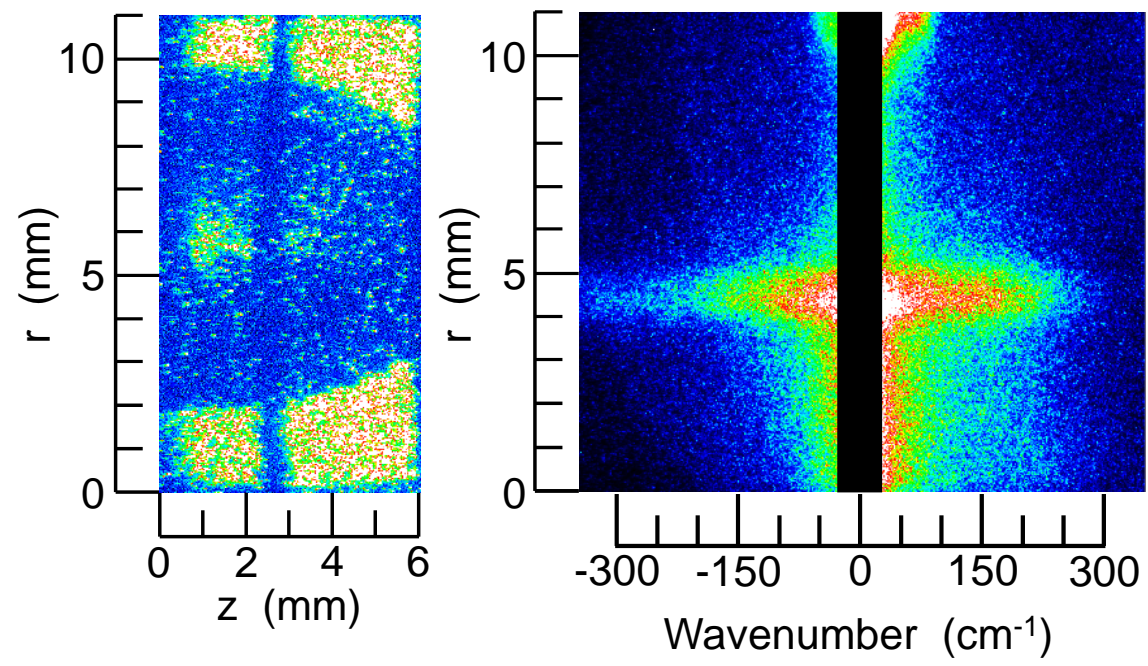

(b) $\mathrm{t}=200 \mu \mathrm{s}$
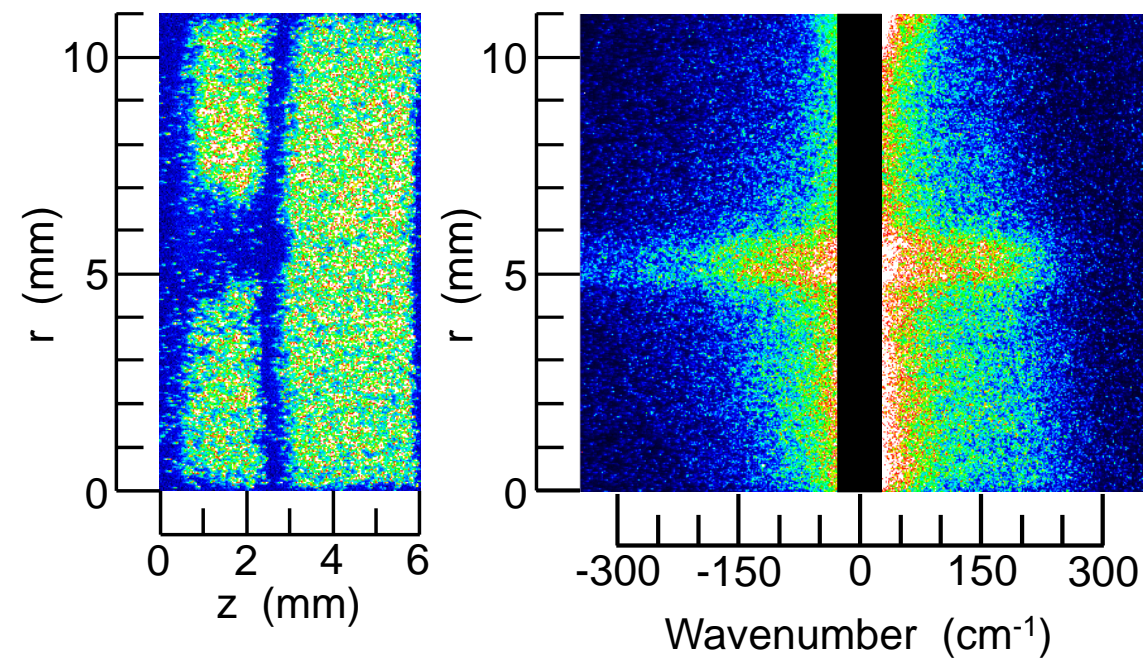

(c) $\mathrm{t}=800 \mu \mathrm{s}$

Fig. 3 Shadowgraph images (left figures) and spatially resolved spectra of scattered laser light (right figures) observed at delay times of (a) $100 \mu \mathrm{s}$, (b) $200 \mu \mathrm{s}$, and (c) $800 \mu \mathrm{s}$. 
the cavitation bubble. However, the scattered laser light from the inside of the cavitation bubble had no wavelength-shifted component. The scattered laser light with no wavelength shift was eliminated by the Rayleigh brock and it was not seen in the spectrum shown in Fig. 3(a).

On the other hand, at a delay time of $200 \mu \mathrm{s}$, which roughly corresponded to the maximum size of the cavitation bubble, we observed the spectrum shown in Fig. 3(b). As shown in the figure, the scattered laser light observed from the inside of the cavitation bubble had the wavelength-shifted component. The wavelength-shifted component had a broader spectral distribution than the Raman scattering of water. The spectrum showing the Raman scattering of water was observed at $2 \lesssim r \lesssim 3 \mathrm{~mm}$ in Fig. 3(b), even though this region corresponded to the inside of the cavitation bubble. This inconsistency was probably caused by the imperfect focusing (collimation) of incident laser beam and the scattered laser light. A similar spectrum to Fig. 3(b) was observed at a delay time of $800 \mu \mathrm{s}$ as shown in Fig. 3(c). The bubble observed at this delay time was a rebounded one after the collapse. The spectrum of the scattered laser light observed from the inside of the cavitation bubble at $800 \mu$ s also had a broader spectral distribution than the Raman scattering of water.

Figure 4 shows a typical spectrum of the scattered laser light, which was obtained by integrating the image shown in Fig. 3(b) along the vertical direction within the range indicated by the arrow. The locations of the Raman peaks of $\mathrm{TiO}_{2}$ corresponding to the rutile, anatase, and brookite crystal structures are indicated in Fig. 4 by the vertical dotted lines. In addition, the arrows with labeled numbers indicate the locations of the peaks shown in Fig. 5, which is the Raman spectrum of nanoparticles collected after the scattering experiment. As shown in Fig. 4, the spectrum of the scattered laser light had no remarkable peaks. The spectrum was roughly independent of the delay time, and the image shown in Fig. 3(c) resulted in a similar spectrum to that shown in Fig. 4.

We observed the synthesis of nanoparticles in water after the scattering experiment. We enriched the colloidal solution of nanoparticles using a centrifugal separator, and sampled the enriched solution from the bottom part. We dripped the droplets with nanoparticles onto a glass substrate, and examined its Raman spectrum using a commercial Raman spectrometer. The Raman spectrum thus obtained is shown in Fig. 5. The locations of the Raman peaks corresponding to rutile, anatase, and brookite $\mathrm{TiO}_{2}$ are indicated by the vertical dotted lines. As shown in Fig. 5, nanoparticles collected after the scattering experiment had a different Raman spectrum from Fig. 4. The three peaks which are labeled with 1-3 are included in the observed wavelength range in the in-situ experiment, and are indicated by arrows in Fig. 4.

\section{Discussion}

As shown in Figs. 3 and 4, we observed spectra which were different from the Raman scattering of water from the region corresponding to the cavitation 


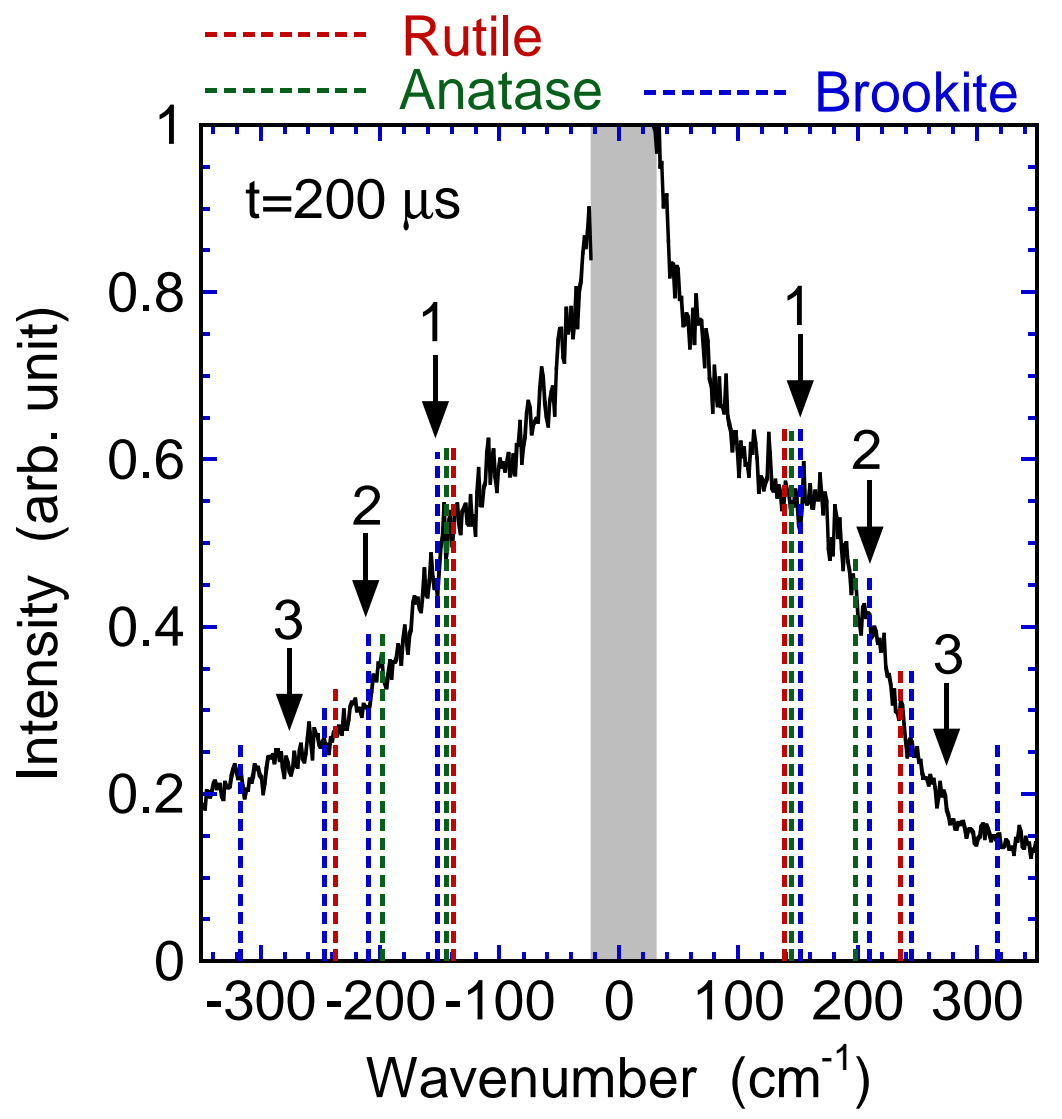

Fig. 4 Typical spectrum of scattered laser light, which was obtained by integrating the image shown in Fig. 3(b) along the vertical direction in the range indicated by the arrow.

bubble. At a delay time of $100 \mu$ s after laser ablation, as shown in Fig. 3(a), the wavelength of the scattered laser light was concentrated at the laser wavelength, and we observed the negligible wavelength-shifted component in the scattered laser light. This result indicates that nanoparticle stored inside the cavitation bubble is not Raman-active at this delay time. A possibility is that nanoparticles are metallic at the short delay time. On the other hand, as shown in Figs. 3(b) and Fig. 4, the scattered laser light from the cavitation bubble had the wavelength-shifted component at a delay time of $200 \mu \mathrm{s}$. This suggests the qualitative change of nanoparticles from metallic to oxidized ones with the delay time after ablation. The reason for the broadened scattered laser light from the limited radial region of $3 \lesssim r \lesssim 6 \mathrm{~mm}$ is unknown. Further experiments with finer focusing into the inside of the cavitation bubble are necessary to know the spatial distribution of the density of oxidized nanoparticles. At a longer delay time of $800 \mu \mathrm{s}$, as shown in Fig. 3(c), the wavelength-shifted 


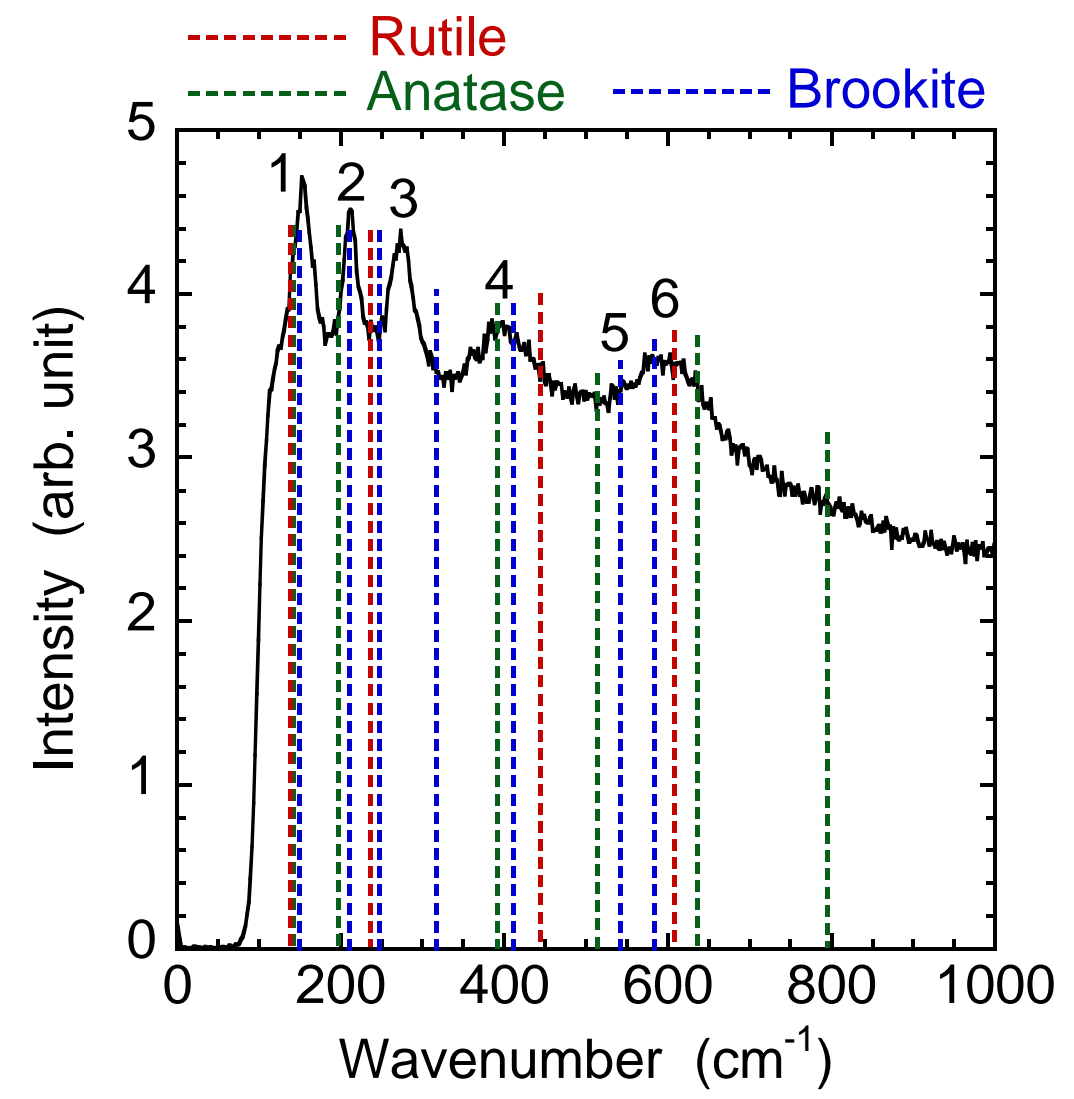

Fig. 5 Raman spectrum of nanoparticles which were collected after the scattering experiment.

component was also observed from the outside of the cavitation bubble. This is because a part of nanoparticles in the cavitation bubble are ejected into water in the expansion phase and at the collapses $[4,12]$. Therefore, water around the rebounded cavitation bubble contains ejected nanoparticles which are detected as the wavelength-shifted component in this experiment.

The objective of the present work was to understand the temporal evolution of nanoparticles from the qualitative point of view. The objective was partly achieved as shown in Figs. 3(a) and 3(b), which suggest the change of nanoparticles from metallic to oxidized ones. However, we did not detect the structural change of nanoparticles in this in-situ experiment. No clear peaks are observed in the spectrum shown in Fig. 4, suggesting that nanoparticles inside the cavitation bubble at $200 \mu$ s after ablation have amorphous structures. The weak humps around +170 and $-150 \mathrm{~cm}^{-1}$, which are close to the Raman peaks of rutile, anatase, and brookite crystals, would be a symptom of the formation of crystalline structures, but no other humps and peaks were 
observed at the wavelengths corresponding to the Raman peaks of crystalline $\mathrm{TiO}_{2}$. Another possibility for the weak hump around $+170 \mathrm{~cm}^{-1}$ is the Raman scattering caused by water droplets inside the cavitation bubble. However, this is unlikely, since water droplets cannot survive inside the cavitation bubble because of the pressure which is lower than the atmospheric pressure $[10,26]$. In contrast to the spectrum shown in Fig. 4, nanoparticles collected after the scattering experiment had clear Raman peaks, as shown in Fig. 5. The present experimental results suggest that the structural change from amorphous to crystalline needs a long time in the synthesis of nanoparticles by liquid-phase laser ablation.

Finally, we briefly discuss the crystal structure of titanium-oxide nanoparticles synthesized in this experiment. The peak labeled with "1" in Fig. 5 is very close to the Raman peak of brookite $\mathrm{TiO}_{2}$. The peak labeled with "2" coincides well with brookite $\mathrm{TiO}_{2}$, while the peak labeled with " 3 " has no agreement with the known Raman peaks of crystalline $\mathrm{TiO}_{2}$. The peak labeled with " 4 " coincides with anatase $\mathrm{TiO}_{2}$, but brookite $\mathrm{TiO}_{2}$ is located within the FWHM of peak "4". The small peak labeled with "5" coincides with brookite $\mathrm{TiO}_{2}$, and the peak labeled with "6" coincides with brookite and rutile $\mathrm{TiO}_{2}$. Accordingly, the synthesis of brookite $\mathrm{TiO}_{2}$ is suggested by the Raman spectrum shown in Fig. 5. However, since we cannot find peaks around 247 and $316 \mathrm{~cm}^{-1}$, which are the locations of the Raman peaks of brookite $\mathrm{TiO}_{2}$, further investigations are necessary to determine the crystal structure of nanoparticles synthesized by the present experiment.

\section{Conclusions}

We carried out in-situ laser-light scattering spectroscopy to understand the qualitative evolution of nanoparticles in liquid-phase laser ablation. The spectrum of the laser light scattered by nanoparticles in a cavitation bubble was measured using a triple-grating spectrograph at various delay times after laser ablation. The spectrum observed at $100 \mu$ s after laser ablation had no wavelength-shifted component, while the scattered laser light with the wavelength-shifted component was observed at a delay time of $200 \mu \mathrm{s}$. This suggests the change from metallic nanoparticles to oxidized ones with the delay time after ablation. We observed no peaks in the spectrum of the scattered laser light in this in-situ experiment, while clear peaks were observed in the Raman spectrum of synthesized nanoparticles. The present experimental results suggest that the crystallization of nanoparticles occurs slowly in liquid.

Acknowledgements This work was supported by a Grant-in-Aid for Scientific Research on Innovative Areas "Frontier Science of Interactions between Plasmas and Nano-Interfaces" (No. 21110004) from the Ministry of Education, Culture, Sports, Science and Technology, Japan. 


\section{References}

1. F. Mafune, J. Kohno, Y. Takeda, T. Kondow, and H. Sawabe, J. Phys. Chem. B 104, $9111(2000)$

2. F. Mafune, J. Kohno, Y. Takeda, T. Kondow, and H. Sawabe, J. Phys. Chem. B 105, 5114 (2001).

3. S. Barcikowski and G. Compagnini, Phys. Chem. Chem. Phys. 15, 3022 (2013)

4. K. Sasaki and N. Takada, Pure Appl. Chem. 82, 1317 (2010)

5. K. Saito, T. Sakka, and Y. H. Ogata, J. Appl. Phys. 94, 5530 (2003)

6. H. Ushida, N. Takada, and K. Sasaki, J. Phys. Conf. Series 59, 563 (2007)

7. T. Tsuji, Y. Tsuboi, N. Kitamura, and M. Tsuji, Appl. Surf. Sci. 229, 365 (2004).

8. Y. Kawaguchi, X. Ding, A. Narazaki, T. Sato, and H. Niino, Appl. Phys. A 79, 883 (2004)

9. K. Sasaki, T. Nakano, W. Soliman, and N. Takada, Appl. Phys. Express 2, 046501 (2009)

10. W. Soliman, T. Nakano, N. Takada, and K. Sasaki, Jpn. J. Appl. Phys. 49, 116202 (2010)

11. C. Liang, Y. Shimizu, M. Masuda, T. Sasaki, and N. Koshizaki, Chem. Mater. 16, 963 (2004)

12. W. Soliman, N. Takada, and K. Sasaki, Appl. Phys. Express 3, 035201 (2010)

13. S. Ibrahimkutty, P. Wagener, A. Menzel, A. Plech, and S. Barcikowski, Appl. Phys. Lett. 101, 103104 (2012).

14. P. Wagener, S. Ibrahimkutty, A. Menzel, A. Plech, and S. Barcikowski, Phys. Chem. Chem. Phys. 15, 3068 (2013)

15. W. Soliman, N. Takada, and K. Sasaki, Jpn. J. Appl. Phys. 50, 108003 (2011)

16. W. Soliman, N. Takada, N. Koshizaki, and K. Sasaki, Appl. Phys. A, 110, 779 (2013)

17. N. Takada, Fujikawa, N. Koshizaki, and K. Sasaki, Appl. Phys. A, 110, 835 (2013)

18. A. De Giacomo, M. Dell'Aglio, A. Santagata, R. Gaudiuso, O. De Pascale, P. Wagener, G. C. Messina, G. Compagnini, and S. Barcikowski, Phys. Chem. Chem. Phys. 15, 3083 (2013)

19. J. Lam, D. Amans, F. Chaput, M. Diouf, G. Ledoux, N. Mary, K. Masenelli-Varlot, V. Motto-Ros, and C. Dujardin, Phys. Chem. Chem. Phys. 16, 963 (2014)

20. M. Dell'Aglio, R. Gaudiuso, O. De Pascale, and A. De Giacomo, Appl. Surf. Sci. 348, $4(2015)$

21. K. Sasaki1, S. Soma, H. Akashi, M. ElSabbagh, and Y. Ikeda, Contrib. Plasma Phys. 55, $563(2015)$

22. A. Kono and K. Nakatani, Rev. Sci. Instrum. 71, 2761 (2000)

23. K. Muraoka and A. Kono, J. Phys. D 44, 043001 (2011)

24. K. Mizoguchi, Y. Hori, and Y. Tominaga, J. Chem. Phys. 97, 1961 (1992)

25. Y. Tominaga and S. M. Takeuchi, J. Chem. Phys. 104, 7377 (1996)

26. C. E. Brennen, Cavitation and Bubble Dynamics (Oxford University Press, New York, 1995) 